\title{
Beneficios de los compuestos bioactivos del mangostán en la enfermedad de Alzheimer: Revisión narrativa
}

\author{
Benefits of bioactive compounds of mangosteen in Alzheimer's disease: \\ narrative review
}

Inmaculada Navarro-González ${ }^{*}$, Pedro Andreo-Martínez², Nuria García-Martínez ${ }^{3}$

\begin{abstract}
Citación/Citation: Navarro-González I, AndreoMartínez P, García-Martínez N. Rev Dis Cli Neuro. 2021; (8)1: 28-38. Available from:

https://doi.org/10.14198/DCN.19622
\end{abstract}

Recibido/Received: 10/04/2021

Aceptado/Accepted: 20/04/2021

Financiación/Funding: Este estudio no ha recibido financiación.

Conflicto de intereses/Conflicts of Interest: Los autores declaran no tener conflicto de intereses.

\section{2021 Inmaculada Navarro-González , Pedro Andreo-Martínez y Nuria García-Martínez}

Licencia/Licence: This work is licensed under a Creative Commons Attribution 4.0 International License (CC BY 4.0).

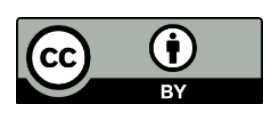

Editorial: Universidad de Alicante (España) Autores/Authors:

1 Área Científica y Técnica de Investigación (ACTI), Sección de Biología Molecular. Universidad de Murcia. España, https://orcid.org/0000-00025894-6400

${ }^{2}$ Departamento de Química Agrícola, Geología y Edafología. Universidad de Murcia. España, https://orcid.org/0000-0001-9028-231X

${ }^{3}$ Departamento de Química Agrícola, Geología y Edafología. Universidad de Murcia. España, https://orcid.org/0000-0001-6535-5492

* Contact for correspondence: inmaculada.navarro@um.es
Resumen: La enfermedad de Alzheimer (EA) es una enfermedad neurodegenerativa irreversible, acompañada de pérdida de memoria y de déficit neurocognitivo progresivo. Los cambios fisiopatológicos asociados a esta enfermedad son la formación extracelular de depósitos de placas del péptido insoluble $ß$-amiloide, la hiperfosforilación de la proteína tau formando ovillos neurofibrilares, además de neuroinflamación, estrés oxidativo, alteración del metabolismo energético, pérdida de neuronas y sinapsis en el cerebro. La patogénesis de la EA no es conocida en su totalidad porque es multifactorial. Por lo tanto, no existe un tratamiento preventivo exitoso. En este sentido, existe un reciente interés sobre los compuestos bioactivos presentes en productos naturales y hierbas medicinales para el tratamiento de la EA por sus efectos beneficiosos. El mangostán, Garcinia mangostana L (familia de Guttifereae), es un árbol tropical que produce una fruta comestible con un sabor dulce y picante; cuyo pericarpio es una fuente de compuestos bioactivos, que han mostrado una excelente actividad farmacológica. El pericarpio del mangostán se ha utilizado en la medicina tradicional contra varias enfermedades como úlceras, infecciones de la piel, diarrea o heridas. Muchos estudios han reportado que el pericarpio del mangostán contiene compuestos fenólicos, vitaminas (B1, B2, C) y otras sustancias bioactivas. Las xantonas y sus derivados pertenecen a la familia de los polifenoles, y están muy presentes en el mangostán. Se ha demostrado que poseen una amplia actividad biológica como antioxidantes, antiinflamatorios, anticancerígenos, antimicrobianos y actividades neuroprotectoras tanto en estudios in vitro como in vivo. La evidencia científica sugiere que las xantonas (obtenidas de pericarpio del mangostán) pueden atenuar la neurotoxicidad y algunos de los cambios fisiopatológicos asociados con la EA en modelos celulares y animales. En la presente revisión, se describe el estado actual del potencial efecto terapéutico del extracto del mangostán y las xantonas en modelos celulares y animales en la EA, describiendo también los efectos observados y las vías moleculares propuestas.

Palabras clave: Compuestos bioactivos; Compuestos neuroprotectores; Enfermedad de Alzheimer; Mangostán; Mangostín. 


\begin{abstract}
Alzheimer's disease (AD) is an irreversible neurodegenerative disease, accompanied by memory loss and progressive neurocognitive deficit. The pathophysiological changes associated with this disease are the extracellular formation of plaque deposits of the insoluble $B$-amyloid peptide, the hyperphosphorylation of the tau protein forming neurofibrillary tangles, in addition to neuroinflammation, oxidative stress, alteration of energy metabolism, loss of neurons and synapses in brain. The pathogenesis of $\mathrm{AD}$ is not fully known because it is multifactorial. Therefore, there is no successful preventive treatment. In this sense, there is a recent interest in the bioactive compounds present in natural products and medicinal herbs for the treatment of $A D$ due to their beneficial effects. The mangosteen, Garcinia mangostana L (Guttifereae family), is a tropical tree that produces an edible fruit with a sweet and spicy flavor; whose pericarp is a source of bioactive compounds, which have shown excellent pharmacological activity. The mangosteen pericarp has been used in traditional medicine against various diseases such as ulcers, skin infections, diarrhea or wounds. Many studies have reported that the mangosteen pericarp contains phenolic compounds, vitamins (B1, B2, C) and other bioactive substances. Xanthones and their derivatives belong to the polyphenol family, and are present in mangosteen. They have been shown to possess broad biological activity as antioxidants, antiinflammatory, anticancer, antimicrobial, and neuroprotective activities in both in vitro and in vivo studies. Scientific evidence suggests that xanthones (obtained from mangosteen pericarp) may attenuate neurotoxicity and some of the pathophysiological changes associated with $\mathrm{AD}$ in both cell and animal models. In the present review, we describe the current status of the potential therapeutic effect of mangosteen extract and xanthones in cell and animal models in AD, in addition to describing the observed effects and the proposed molecular pathways.
\end{abstract}

Keywords: Alzheimer's disease; Bioactive compounds; Mangosteen; Mangostin; Neuroprotective compounds.

\title{
1. Introducción
}

Garcinia mangostana Linn es un árbol de origen asiático que produce una fruta denominada mangostán o mangostino, conocida como la reina de las frutas. El mangostán pesa entre 80 y 140 g y consta de tres partes anatómicas bien diferenciadas: el pericarpio o corteza, la pulpa (que es la parte comestible) y dos o tres semillas [1]. Durante muchos años, el pericarpio de esta fruta ha sido empleado en la medicina tradicional para tratar procesos inflamatorios, ulceras, infecciones cutáneas y heridas, disentería, diarreas, etc. [2]. Se tiene constancia científica de que las funciones biológicas antiinflamatorias, antioxidantes, antitumorales, antibacterianas y neuroprotectoras atribuidas a esta fruta, principalmente al pericarpio, se deben a que es una excelente fuente de sustancias químicas biológicamente activas [3]. Comparando la parte comestible de esta fruta con el pericarpio, este último contiene 10 veces más compuestos fenólicos y una actividad antioxidante 20 veces mayor [4]. Actualmente se han identificado parte de los compuestos bioactivos de esta fruta, siendo las xantonas y sus derivados los que están en mayor cantidad y a las que se les ha atribuido la mayoría de sus propiedades beneficiosas [5]. Las xantonas son unos metabolitos secundarios de las plantas, de naturaleza fenólica, con una estructura química compuesta por un anillo tricíclico; y grupos metilo, hidroxilo o isopreno unidos a los anillos de su estructura, que les proporciona 
gran versatilidad (ver Figura 1). Al menos 68 xantonas distintas han sido identificadas en esta fruta, y más de 50 están presentes en el pericarpio en altas concentraciones. De todas las xantonas identificadas, las más estudiadas son la $\alpha$-mangostín y $\beta$-mangostín, aunque también se han encontrado otras como $\gamma$ mangostín, gartanina, 3-isomangostín, garcinonas A, B, C, D y E, 8-deoxigartanina, mangostanol, isomangostina y garciniafuranona [6]. En este sentido, diversos estudios científicos, in vitro e in vivo, han demostrado que las xantonas del mangostán muestra un efecto neuroprotector frente la enfermedad de Alzheimer (EA) [2].

Figura 1. Estructura química de algunas xantonas identificadas en el mangostán.<smiles>O=c1c2ccccc2oc2ccccc12</smiles>

Xantona, estructura básica<smiles>CC(C)=CCc1c(O)c(CC=C(C)C)c2oc3c(O)cccc3c(=O)c2c1O</smiles>

8-deoxigartanina<smiles>CC(C)=CCc1c(O)cc2oc3cc(O)c(O)c(CC=C(C)C)c3c(=O)c2c1O</smiles>

Gamma-mangostín<smiles>COc1c(O)cc2oc3cc4c(c(O)c3c(=O)c2c1CC=C(C)C)CCC(C)(C)O4</smiles>

3-isomangostín

La enfermedad de Alzheimer es la más común de las enfermedades neurodegenerativas. El primero en describir la enfermedad fue el psiquiatra Alöis Alzheimer en 1907, después de detectarla en una fémina de 51 años. Tras el fallecimiento de la paciente se le realizó un estudio microscópico a su cerebro, detectando placas seniles, ovillos neurofibrilares y presencia de la proteína ß-amiloide ( $\beta A)$. En la actualidad, estos son los rasgos neuropatológicos que se asocian a la EA [7].

Esta revisión se centra en entender la etiología de la EA, para, posteriormente, describir y comprender los efectos neuroprotectores de los compuestos bioactivos presentes en el mangostán; descritos hasta la actualidad. Todo ello redactado en español para facilitar la difusión de las propiedades beneficiosas para la salud de este alimento, principalmente sobre la EA, en la comunidad científica y en el resto de la población de habla hispana.

\section{Método}

El presente artículo es un estudio de revisión narrativa, que se centra en la descripción de estudios científicos que han evaluado los compuestos bioactivos presentes en el pericarpio del mangostán y su prometedor efecto neuroprotector sobre la enfermedad de Alzheimer. 
La revisión bibliográfica se realizó utilizando las bases de datos especializadas: Pubmed, Scopus y Web of Science, incluyendo todos los artículos publicados hasta el año 2020. Los términos utilizados en la búsqueda fueron: “Garcinia mangosteen”, “Alzheimer`s disease”, “degeneratives diseases”, "neuroprotective effect mangosteen" y distintas combinaciones de ellos. Los criterios de inclusión fueron que los artículos estuvieran publicados en revistas científicas indexadas en alguna base de datos científica. Los idiomas que se prefirieron fueron el inglés y el español, excluyendo artículos redactados en otras lenguas. La búsqueda de la información se realizó en enero de 2021. Finalmente, tras la lectura de los resúmenes de los artículos, se seleccionaron aquellos artículos que se centraban en las áreas de investigación de interés para la presente revisión bibliográfica.

\section{Resultados y Discusión}

\subsection{Etiología y fisiopatología de la enfermedad}

La EA es una enfermedad neurodegenerativa cuya progresión es lenta, y se sabe que comienza muchos años antes del inicio de los síntomas. Las características neuropatológicas aceptadas en la clínica de la enfermedad son: (I) la formación y la deposición de placas extracelulares del péptido insoluble $\beta$ A y (II) la hiperfosforilación de la proteína tau asociada a microtúbulos, formando los ovillos neurofibrilares [8]. A pesar de que las características patológicas están muy bien definidas, la etiología de la enfermedad es desconocida por el momento.

La placa intracelular $\beta \mathrm{A}$ es un péptido corto, cuyas aloformas más comunes son $\beta \mathrm{A} 1-40$ y $\beta \mathrm{A} 1-42$, derivadas de la escisión secuenciada de la proteína precursora del amiloide (APP) por las denominadas secretasas $\beta$ y $\gamma[9,10]$. Como consecuencia de estas escisiones, los péptidos A y $\beta$ se agregan y se depositan, extracelularmente, en placas seniles difusas $[8,11]$. Este proceso interfiere en la sinapsis entre las neuronas, lidera la neurotoxicidad producida en la EA y conduce a la muerte de las células neuronales [8].

La proteína tau está involucrada en el ensamblaje y estabilización de microtúbulos en neuronas [12]. Sin embargo, la hiperfosforilación de la proteína tau causa la pérdida de la estabilización de los microtúbulos, lo que conduce a una anormalidad en la forma y funcionalidad de las neuronas, provocando finalmente la muerte de éstas $[13,14]$.

Por otro lado, tanto los depósitos $\beta$ A como la proteína tau hiperfosforilada desestabilizan la homeostasis del calcio y aumenta la vulnerabilidad de las neuronas a la excitotoxicidad, principalmente a través de interacciones con receptores de N-metil-D-aspartato (NMDA) [15, 16].

Los procesos descritos en las líneas anteriores inducen el deterioro en la función cognitiva y de la memoria, síntomas observados en pacientes con EA [13, 17]. Además, la deficiencia intelectual observada en pacientes con EA se ha relacionado con una pérdida de la función colinérgica en determinadas partes anatómicas especificas del cerebro [18]. Los inhibidores colinérgicos, principalmente de la acetilcolinesterasa (AChe), son empleados para mantener los niveles de acetilcolina y mantener la sinapsis. Asimismo, la EA tiene asociadas otras patologías como son la depresión, la neuroinflamación, el estrés oxidativo, la disfunción mitocondrial y reducción de factores neurotróficos como el factor neurotrófico derivado del cerebro (BDNF). La neuroinflamación consiste en la activación de la microglía (células del 
sistema inmune innato del sistema nervioso central) en respuesta a la perturbación de la homeostasis. La activación de la microglía provoca la liberación de citoquinas proinflamatorias [19].

El estrés oxidativo es otra característica común de la EA. Las especies reactivas de oxígeno o radicales libres (ROS) producidos por el estrés oxidativo afectan a la estructura y función de las células neuronales, contribuyendo al desarrollo de la EA [20,21]. Se sabe que el cerebro es susceptible de sufrir estrés oxidativo por la deficiencia de antioxidantes endógenos, por contener altos niveles de lípidos insaturados susceptibles de ser oxidados y una elevada tasa de utilización de oxígeno. Además, $\beta \mathrm{A}$ induce al daño oxidativo de las neuronas por la generación de ROS [4].

Por su relación directa con el estrés oxidativo, la disfunción mitocondrial también juega un papel clave en la patogenia de la EA [22], lo que finalmente desencadena en una apoptosis [8]. Aunque la apoptosis es un proceso normal y necesario (porque elimina células dañadas de forma irreversible o innecesarias para evitar su propagación), un exceso de apoptosis da lugar a las llamadas enfermedades neurodegenerativas, donde las células mueren antes de tiempo ya que reciben una señal que las induce a destruirse.

La falta o escasez de determinados factores de crecimiento pueden ser causa de los procesos neurodegenerativos. El factor de crecimiento nervioso (NGF), la neurotrofina 3 (NT-3) o el BDNF son miembros de la familia genética de las neurotrofinas, que inducen supervivencia, diferenciación, mantenimiento y reparación de poblaciones neuronales específicas. Por tanto, el NGF, la NT-3, y especialmente, BDNF son cruciales para la supervivencia, el mantenimiento y la regeneración de neuronas específicas en el cerebro adulto [23]. Y se han observado reducciones significativas de estos factores neurotróficos en la EA [23, 24], así como en la depresión [19].

Se sabe que hay mutaciones genéticas, principalmente de los genes que codifican la APP, que favorecen la aparición de la enfermedad en edades tempranas. Estos genes cumplen una herencia autosómica dominante y han sido asociados a la EA familiar. La demencia tipo EA parece ser el resultado terminal de una interacción compleja que incluye factores genéticos, cambios atribuibles a múltiples patologías subyacentes, trauma, injurias ambientales, y cambios hormonales entre muchos otros [7].

\subsection{Efecto neuroprotector de las xantonas del mangostán sobre la EA}

El potencial del mangostán para atenuar la neurotoxicidad y algunos cambios fisiopatológicos asociados con la EA como la neuroinflamación, la agregación del péptido $\beta$ A y las alteraciones de la memoria han sido estudiadas por la comunidad científica.

Tabla 1. Principales resultados encontrados en los estudios seleccionados en la presente revisión narrativa.

\begin{tabular}{|c|c|c|c|c|}
\hline Referencia & $\begin{array}{l}\text { Extracto } \\
\text { compuesto }\end{array}$ & $\mathbf{o}$ & Modelo animal & Resultados principales \\
\hline \multirow[t]{2}{*}{ [25] } & EPM & & Línea celular & $\uparrow$ Actividad neuroprotectora \\
\hline & & & NG108-15 & $\uparrow$ Actividad antioxidante \\
\hline \multirow[t]{3}{*}{ [5] } & EPM & & Línea celular & $\downarrow$ Neurotoxicidad \\
\hline & & & SK-N-SH & $\downarrow$ Caspasa-3 \\
\hline & & & & $\downarrow$ ROS \\
\hline
\end{tabular}


[26]

EPM

[27]

[29]

[30]

EPM

EPM

EPM

[31]

[32]

[3]

EPM dieta

Línea celular

SK-N-SH

$\alpha$-mangostín

EPM dieta

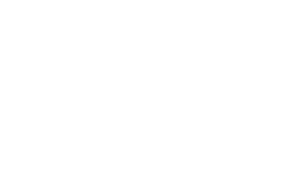

$\alpha$-mangostín

$\alpha$-mangostín

$\alpha$-mangostín,

$\gamma$-mangostín,

Gartanina,
Ratón B6

Ratón 3Xtg-AD

Ratones SA macho $\downarrow$ Neurotoxicidad oxidativa

$\downarrow$ Caspasa-3

$\downarrow$ ROS

$\downarrow$ Actividad AChE

$\downarrow$ Deterioro de la memoria

$\downarrow$ Caspasa-3

$\downarrow$ ROS en el cerebro

$\downarrow$ Deterioro de la memoria

$\downarrow R O S$ en el cerebro

Actividad AChE restaurada

Cultivo primario de células neuronales corticales de rata

Línea celular OHSC

$\downarrow$ Neurotoxicidad

$\uparrow$ niveles de BDNF

$\uparrow$ niveles de BDNF

$\downarrow$ IL6

$\downarrow$ Deposición de tau fosforilada

$\downarrow$ Deterioro cognitivo

$\downarrow$ Deposición de placas $\beta \mathrm{A}$

$\downarrow$ Deposición de tau fosforilada

$\downarrow$ Deterioro de la memoria

$\downarrow$ Deterioro cognitivo y de la memoria

$\downarrow$ Niveles de AChE

$\uparrow$ Actividad antioxidante endógena

$\downarrow$ IL6, COX-2 (neuroinflamación)

$\downarrow$ Producción de placas $\beta$ A

$\downarrow$ Actividad de secretasas $\beta$ y $\gamma$

células neuronales corticales de rata

$\downarrow$ Neurotoxicidad

$\downarrow$ ROS

$\downarrow$ Producción de placas $\beta \mathrm{A}$

Garcinona C 
[33] $\gamma$-mangostín

EPM

[34]
Cultivo primario de células neuronales corticales de rata

Células neuronales

Ratones ICR

Cultivo primario de células neuronales corticales de rata $\downarrow$ Neurotoxicidad oxidativa

$\downarrow$ Caspasa-3 y 9

$\downarrow$ ROS

$\downarrow$ Peroxidación lipídica

$\downarrow$ Actividad $\beta$-secretasa

$\downarrow$ Deterioro de la memoria

$\downarrow$ Neurotoxicidad

$\downarrow$ ROS

$\downarrow$ Caspasa-3

$\downarrow$ Peroxidación lipídica

$\downarrow$ Actividad $\beta$-secretasa

$\downarrow$ Actividad AChE

Ratones ICR

Mejora de la memoria

Hiperperfusión

cerebral en Ratas

Hiperperfusión

cerebral en Ratas $\downarrow$ Deterioro cognitivo

$\downarrow$ Deterioro cognitivo,

Mejora memoria y aprendizaje

Nota: EPM=Extracto obtenido del pericarpio del mangostán; Ratones ICR= Institute of Cancer Research; 3xTg-AD= Ratón transgénico para la enfermedad de Alzheimer; BDNF= Factor neurotrófico derivado del cerebro; $\beta$ A=Péptido $\beta$-amiloide; $A C h E=$ Acetilcolinesterasa; IL-6= Interleucina-6; ROS= Especies reactivas del oxígeno

Se han encontrado varios estudios científicos, in vitro e in vivo, en los que se evalúa el efecto beneficioso de extractos obtenidos del pericarpio del mangostán sobre la enfermedad de Alzheimer. Y en otros estudios se ha evaluado el efecto de algunas xantonas específicas identificadas en los extractos (véase Tabla 1).

En el año 2006, Weecharangsan, Opanasopit [25] estudiaron el efecto neuroprotector de 4 extractos, obtenidos del pericarpio del mangostán de forma diferente, en células de neuroblastoma. Estos investigadores concluyeron que los 4 extractos exhibieron actividad antioxidante, pero el extracto acuoso y el que contenía un $50 \%$ de etanol fueron los que mayor actividad antioxidante y efecto neuroprotector mostraron, sugiriendo su posible aplicación en enfermedades neurodegenerativas como la EA.

Posteriormente, Moongkarndi, Srisawat [5], en el año 2010, estudiaron el efecto protector de un extracto obtenido del pericarpio del mangostán contra la citotoxicidad inducida por la $\beta A 1-42$, encontrando una reducción significativa en los niveles de ROS y de la actividad de la caspasa-3 (mediador esencial de la muerte celular programada: apoptosis) en células de neuroblastoma (SK-N-SH). Y, curiosamente, se 
observaron cambios en la expresión de algunas proteínas como la proteína 4 de unión a FK506 y la proteína BAT1, sugiriendo que estas son las proteínas diana de los compuestos bioactivos presentes en el extracto.

Un extracto de mangostán con elevadas cantidades de polifenoles y, por ende, una elevada actividad antioxidante, antagonizó los efectos del $\mathrm{H}_{2} \mathrm{O}_{2}$ y bifenilos policlorados sobre la viabilidad celular, en la producción de ROS y actividad sobre la caspasa-3 en células SK-N-SH. El extracto también mostró una mejora en la disfunción de la memoria inducida por escopolamina en ratones. Además, un aumento de la actividad de los niveles de ROS y caspase-3 en el cerebro de ratones tratados con escopolamina fueron inhibidos por el tratamiento con el extracto [26].

Por otro lado, Phyu and Tangpong [27] evidenciaron en el año 2014 que ratones alimentados diariamente con un extracto acuoso obtenido del pericarpio del mangostán provocó mejoras en la pérdida de memoria y en la depresión. Este extracto, rico en xantonas, también fue capaz de restaurar la actividad ACheE y una atenuación de los ROS en el cerebro.

En otra serie de experimentos se describió la capacidad de $\alpha$-mangostín para atenuar la neurotoxicidad inducida por la $\beta \mathrm{A}$ en las neuronas corticales cerebrales primarias de ratas (sello distintivo de la aparición y progresión) [28]. Estos datos fueron apoyados por Huang, Chen [29], los cuales hallaron que el tratamiento con un extracto del pericarpio redujo significativamente la muerte celular y aumentó el BDNF en un cultivo de células. Posteriormente, el extracto al que se le identificaron las xantonas, fue administrado en la dieta de ratones adultos durante 8 meses; concluyendo que el extracto atenuaba significativamente el deterioro cognitivo. Por lo que a posteriori, lo administraron a un modelo animal usado para estudiar la EA. Concluyeron que la administración del extracto del pericarpio en la dieta ejerció efectos neuroprotectores, antioxidantes y antiinflamatorios y redujo los niveles de deposición de la placa $\beta A$ y p-tau en el hipocampo de ratones $3 \times \mathrm{Tg}-\mathrm{AD}$, lo que podría atenuar aún más el déficit de recuperación de memoria. En otro estudio en el que igualmente emplearon ratones a los que se les indujo la EA, pero antes de ello se les administró un extracto obtenido del pericarpio del mangostán durante 28 días, también observaron una mejora en la función cognitiva, una reducción de la actividad de la AChE y un incremento de las enzimas antioxidantes endógenas (glutatión, catalasa y peroxidasa) [30].

Otro estudio evaluó que una dosis diaria de $\gamma$-mangostín en ratones era capaz de reducir la neuroinflamación inducida; y los niveles cerebrales de IL-6 y COX-2 también fueron disminuidos (lo que se traduce en una menor inflamación) [31]. Por su parte, Zhao, Wang [32] demostraron que la administración de $\alpha$-mangostín puro disminuye la producción de la placa $\beta$ A a través de la disminución de la actividad de la $\beta$-secretasa y probablemente también de la $\gamma$-secretasa.

En esta línea, una serie de xantonas aisladas del pericarpio del mangostán ( $\alpha$-mangostín, 8-deoxigartanina, gartanina, garciniafuranona, garcinona $\mathrm{C}$, garcinona $\mathrm{D}$ y $\gamma$-mangostín) fueron utilizadas en células de $E$. coli. Los resultados obtenidos indicaron que la mayoría de las xantonas exhibían una inhibición significativa de la agregación de la placa $\beta$ A cuando se inducia y una capacidad moderada para inhibir BACE1, actuando como antioxidantes y quelantes de biometales. Entre estos compuestos, $\alpha$-mangostín, gartanina, garcinona $\mathrm{C}$ y $\gamma$-mangostín mostraron mejores propiedades antioxidantes y potentes efectos neuroprotectores contra la muerte celular inducida en la línea celular HT22 (línea neuronal) a través de un mecanismo mediado por su capacidad de captar radicales libres. Además, gartanina, garcinona $\mathrm{C}$ y $\gamma$ mangostín podrían ser capaces de atravesar la barrera hematoencefálica in vitro. Estos hallazgos sugieren 
que las xantonas naturales tienen actividades multifuncionales contra la EA y podrían ser compuestos prometedores para la terapia de esta enfermedad [3].

Lee, Kim [33] concluyeron que la $\gamma$-mangostín pura, aislada del pericarpio del mangostán, exhibió un potente efecto neuroprotector atribuido a su actividad antioxidante y antiapoptótica (inhibición caspasa 3 y 9). Y tanto la $\gamma$-mangostín como la $\alpha$-mangostín redujeron la actividad de la $\beta$-secretasa cuando se añadían a cultivos primarios. Además, una administración oral en ratones (de 10 y $30 \mathrm{mg} / \mathrm{kg}$ ) mejoraba la memoria.

Un estudio publicado en el año 2021, evaluó el potencial farmacológico de un extracto acuoso obtenido del pericarpio del mangostán en un cultivo primario obtenido de células corticales de rata, encontraron que el extracto aminoraba la neurotoxicidad y la producción de ROS. También presentó acción contra la apoptosis (por la inhibición de la Caspasa 3) y antioxidante (reducción de la peroxidación lipídica). Además, suprimió la actividad de la $\beta$-secretasa y AChe. Por otra parte, los investigadores evaluaron el efecto del extracto sobre la memoria en ratones, sugiriendo que ejerce una mejora sobre la memoria a través de su actividad antioxidante y antiapoptótica [4]. En otro estudio realizado recientemente, donde se analizó la composición de un extracto obtenido del pericarpio, se encontró que la $\alpha$-mangostín estaba presente en un 55,8 \%. Estos investigadores evaluaron el efecto de la $\alpha$-mangostín en ratas y concluyeron que ambos revierten el deterioro cognitivo. Tras estudiar más a fondo la $\alpha$-mangostín también concluyeron que su administración mejoró el déficit de aprendizaje y memoria en tratamientos agudos y subagudos en ratas mejorando el aprendizaje espacial, pero no la potenciación a largo plazo en el hipocampo [34].

\section{Conclusiones}

De todos los estudios científicos revisados, se puede concluir que las diferentes formas de administrar las xantonas del pericarpio del mangostán, extractos a células o en la dieta o de forma aislada, tienen múltiples propiedades farmacológicas y efectos dirigidos a las diferentes patologías que se producen en la EA como en la prevención de la producción de la $\beta$ A (deposición y toxicidad), actividad antioxidante, antiinflamatoria, inhibición de la hiperfosforilación de tau, excitotoxicidad por NMDA y actividad de AChe. Además de la mejora cognitiva y de las funciones de la memoria. Pero todos estos datos han sido evaluados en modelos animales y celulares, por lo que las próximas investigaciones deben ir dirigidas a humanos.

\section{Referencias}

1. Navarro-González I, Codina-Diaz E, Periago MJ. Propiedades beneficiosas para la salud del mangostán. Rev esp nutr comunitaria 2015, 21 (3): 29-37.

2. Wang MH, Zhang KJ, Gu QL, Bi XL, Wang JX. Pharmacology of mangostins and their derivatives: A comprehensive review. Chin J Nat Med 2017, 15 (2): 81-93. doi: https://doi.org/10.1016/s18755364(17)30024-9 
3. Wang SN, Li Q, Jing MH, Alba E, Yang XH, Sabaté R, et al. Natural Xanthones from Garcinia mangostana with Multifunctional Activities for the Therapy of Alzheimer's Disease. Neurochem Res 2016, 41 (7): 1806-1817. doi: https://doi.org/10.1007/s11064-016-1896-y

4. Oh Y, Do HTT, Kim S, Kim Y-M, Chin Y-W, Cho J. Memory-Enhancing Effects of Mangosteen Pericarp Water Extract through Antioxidative Neuroprotection and Anti-Apoptotic Action. Antioxidants 2021, 10 (1): 34. doi: https://doi.org/10.3390/antiox10010034

5. Moongkarndi P, Srisawat C, Saetun P, Jantaravinid J, Peerapittayamongkol C, Soi-ampornkul R, et al. Protective effect of mangosteen extract against beta-amyloid-induced cytotoxicity, oxidative stress and altered proteome in SK-N-SH cells. J Proteome Res 2010, 9 (5): 2076-2086. doi: https://doi.org/10.1021/pr100049v

6. Do HTT, Cho J. Mangosteen Pericarp and Its Bioactive Xanthones: Potential Therapeutic Value in Alzheimer's Disease, Parkinson's Disease, and Depression with Pharmacokinetic and Safety Profiles. Int J Mol Sci 2020, 21 (17). doi: https://doi.org/10.3390/ijms21176211

7. Bondi MW, Edmonds EC, Salmon DP. Alzheimer's Disease: Past, Present, and Future. J Int Neuropsychol Soc 2017, 23 (9-10): 818-831. doi: https://doi.org/10.1017/s135561771700100x

8. Tiwari S, Atluri V, Kaushik A, Yndart A, Nair M. Alzheimer's disease: pathogenesis, diagnostics, and therapeutics. Int J Nanomedicine 2019, 14: 5541-5554. doi: https://doi.org/10.2147/ijn.S200490

9. Salomone S, Caraci F, Leggio GM, Fedotova J, Drago F. New pharmacological strategies for treatment of Alzheimer's disease: focus on disease modifying drugs. Br J Clin Pharmacol 2012, 73 (4): 504-517. doi: https://doi.org/10.1111/j.1365-2125.2011.04134.x

10. Menendez-Gonzalez M, Padilla-Zambrano HS, Alvarez G, Capetillo-Zarate E, Tomas-Zapico C, Costa A. Targeting Beta-Amyloid at the CSF: A New Therapeutic Strategy in Alzheimer's Disease. Front Aging Neurosci 2018, 10: 100-100. doi: https://doi.org/10.3389/fnagi.2018.00100

11. Hardy J. The amyloid hypothesis for Alzheimer's disease: a critical reappraisal. J Neurochem 2009, 110 (4): 1129-1134. doi: https://doi.org/10.1111/j.1471-4159.2009.06181.x

12. Chakravarthy M, Chen S, Dodd PR, Veedu RN. Nucleic Acid-Based Theranostics for Tackling Alzheimer's Disease. Theranostics 2017, 7 (16): 3933-3947. doi: https://doi.org/10.7150/thno.21529

13. Pooler AM, Polydoro M, Wegmann S, Nicholls SB, Spires-Jones TL, Hyman BT. Propagation of tau pathology in Alzheimer's disease: identification of novel therapeutic targets. Alzheimer's Res Ther 2013, 5 (5): 49. doi: https://doi.org/10.1186/alzrt214

14. Kumar K, Kumar A, Keegan RM, Deshmukh R. Recent advances in the neurobiology and neuropharmacology of Alzheimer's disease. Biomed Pharmacother 2018, 98: 297-307. doi: https://doi.org/10.1016/j.biopha.2017.12.053

15. Mattson MP, Cheng B, Davis D, Bryant K, Lieberburg I, Rydel RE. beta-Amyloid peptides destabilize calcium homeostasis and render human cortical neurons vulnerable to excitotoxicity. J Neurosci 1992, 12 (2): 376-389. doi: https://doi.org/10.1523/jneurosci.12-02-00376.1992

16. Couratier P, Lesort M, Sindou P, Esclaire F, Yardin C, Hugon J. Modifications of neuronal phosphorylated $\tau$ immunoreactivity induced by NMDA toxicity. Mol Chem Neuropathol 1996, 27 (3): 259-273. doi: https://doi.org/10.1007/BF02815108

17. Hardy JA, Higgins GA. Alzheimer's disease: the amyloid cascade hypothesis. Science 1992, 256 (5054): 184-185. doi: https://doi.org/10.1126/science.1566067

18. Giacobini E. Cholinergic function and Alzheimer's disease. Int J Geriatr Psychiatry 2003, 18 (Suppl 1): S1-5. doi: https://doi.org/10.1002/gps.935

19. Peng S, Li W, Lv L, Zhang Z, Zhan X. BDNF as a biomarker in diagnosis and evaluation of treatment for schizophrenia and depression. Discov Med 2018, 26 (143): 127-136. 
20. Jiang T, Sun Q, Chen S. Oxidative stress: A major pathogenesis and potential therapeutic target of antioxidative agents in Parkinson's disease and Alzheimer's disease. Prog Neurobiol 2016, 147: 1-19. doi: https://doi.org/10.1016/j.pneurobio.2016.07.005

21. Umeno A, Biju V, Yoshida Y. In vivo ROS production and use of oxidative stress-derived biomarkers to detect the onset of diseases such as Alzheimer's disease, Parkinson's disease, and diabetes. Free Radic Res 2017, 51 (4): 413-427. doi: https://doi.org/10.1080/10715762.2017.1315114

22. Wang X, Wang W, Li L, Perry G, Lee HG, Zhu X. Oxidative stress and mitochondrial dysfunction in Alzheimer's disease. Biochim Biophys Acta 2014, 1842 (8): 1240-1247. doi: https://doi.org/10.1016/j.bbadis.2013.10.015

23. Allen SJ, Watson JJ, Shoemark DK, Barua NU, Patel NK. GDNF, NGF and BDNF as therapeutic options for neurodegeneration. Pharmacol Ther 2013, 138 (2): 155-175. doi: https://doi.org/10.1016/j.pharmthera.2013.01.004

24. Tanila H. The role of BDNF in Alzheimer's disease. Neurobiol Dis 2017, 97: 114-118. doi: https://doi.org/10.1016/j.nbd.2016.05.008

25. Weecharangsan W, Opanasopit P, Sukma M, Ngawhirunpat T, Sotanaphun U, Siripong P. Antioxidative and neuroprotective activities of extracts from the fruit hull of mangosteen (Garcinia mangostana Linn.). Med Princ Pract 2006, 15 (4): 281-287. doi: https://doi.org/10.1159/000092991

26. Sattayasai J, Chaonapan P, Arkaravichie T, Soi-ampornkul R, Junnu S, Charoensilp P, et al. Protective Effects of Mangosteen Extract on H2O2-Induced Cytotoxicity in SK-N-SH Cells and ScopolamineInduced Memory Impairment in Mice. PLOS ONE 2013, 8 (12): e85053. doi: https://doi.org/10.1371/journal.pone.0085053

27. Phyu MP, Tangpong J. Neuroprotective effects of xanthone derivative of Garcinia mangostana against lead-induced acetylcholinesterase dysfunction and cognitive impairment. Food Chem Toxicol 2014, 70: 151-156. doi: https://doi.org/10.1016/j.fct.2014.04.035

28. Wang Y, Xia Z, Xu J-R, Wang Y-X, Hou L-N, Qiu Y, et al. $\alpha$-Mangostin, a polyphenolic xanthone derivative from mangosteen, attenuates $\beta$-amyloid oligomers-induced neurotoxicity by inhibiting amyloid aggregation. Neuropharmacology 2012, 62 (2): 871-881. doi: https://doi.org/10.1016/j.neuropharm.2011.09.016

29. Huang H-J, Chen W-L, Hsieh R-H, Hsieh-Li HM. Multifunctional Effects of Mangosteen Pericarp on Cognition in C57BL/6J and Triple Transgenic Alzheimer's Mice. Evid-Based Compl Alt 2014, 2014: 813672. doi: https://doi.org/10.1155/2014/813672

30. P A, Reddy R, Begum N, Bakshi V. Neuroprotective Effect of Garcinia Mangostana on Streptozotocin Induced Sporadic Type Alzheimer's Disease in Mice. Int J Appl Pharm Sci Res 2016, 1. doi: https://doi.org/10.21477/ijapsr.v1i1.9603

31. Nava Catorce M, Acero G, Pedraza-Chaverri J, Fragoso G, Govezensky T, Gevorkian G. Alphamangostin attenuates brain inflammation induced by peripheral lipopolysaccharide administration in C57BL/6J mice. J Neuroimmunol 2016, 297: 20-27. doi: https://doi.org/10.1016/j.jneuroim.2016.05.008

32. Zhao LX, Wang Y, Liu T, Wang YX, Chen HZ, Xu JR, et al. $\alpha$-Mangostin decreases $\beta$-amyloid peptides production via modulation of amyloidogenic pathway. CNS Neurosci Ther 2017, 23 (6): 526-534. doi: https://doi.org/10.1111/cns.12699

33. Lee Y, Kim S, Oh Y, Kim Y-M, Chin Y-W, Cho J. Inhibition of Oxidative Neurotoxicity and Scopolamine-Induced Memory Impairment by " $\gamma$ "-Mangostin: "In Vitro" and "In Vivo" Evidence. Oxid Med Cell Longev 2019, 2019: 3640753. doi: https://doi.org/10.1155/2019/3640753

34. Tiang N, Ahad M, Murugaiyah V, Hassan Z. Xanthone - enriched fraction of Garcinia mangostana and $\alpha$ - mangostin improve the spatial learning and memory of chronic cerebral hypoperfusion rats. J Pharm Pharmacol 2020, 72. doi: https://doi.org/10.1111/jphp.13345 